Relations industrielles

Industrial Relations

\title{
Les effectifs syndicaux québécois ou quand les chiffres dansent...
}

\section{François Delorme et Gaspar Lassonde}

Volume 34, numéro 2, 1979

URI : https://id.erudit.org/iderudit/028971ar

DOI : https://doi.org/10.7202/028971ar

Aller au sommaire du numéro

Éditeur(s)

Département des relations industrielles de l'Université Laval

ISSN

0034-379X (imprimé)

1703-8138 (numérique)

Découvrir la revue

Citer cet article

Delorme, F. \& Lassonde, G. (1979). Les effectifs syndicaux québécois ou quand les chiffres dansent.... Relations industrielles / Industrial Relations, 34(2),

376-381. https://doi.org/10.7202/028971ar

Tous droits réservés @ C Département des relations industrielles de l'Universite Laval, 1979
Ce document est protégé par la loi sur le droit d'auteur. L'utilisation des services d'Érudit (y compris la reproduction) est assujettie à sa politique d'utilisation que vous pouvez consulter en ligne.

https://apropos.erudit.org/fr/usagers/politique-dutilisation/ 


\section{DIALOGUE}

\section{Les effectifs syndicaux québécois ou quand les chiffres dansent...}

\section{François Delorme \\ et}

\section{Gaspar Lassonde}

Dans le dernier numéro de 1978, vol. 33, no 4, de la revue Relations Industrielles, monsieur Gérard Dion a minutieusement recensé un texte que nous avons publié par l'entremise de la Direction générale de la recherche du ministère du Travail et de la Main-d'oeuvre, alors que nous exerçions tous deux nos fonctions à cette Direction. Ce texte, intitulé Aspects de la réalité syndicale québécoise - 1976, cherche à tracer un portrait des effectifs syndicaux québécois au moyen de divers tableaux descriptifs. Nous savons gré à monsieur Dion d'avoir amorcé un débat - fécond, nous l'espérons - sur ce thème précis et de soulever, dans sa recension, certains problèmes sur lesquels nous aimerions revenir. Nos commentaires porteront sur quatre éléments de la problématique mais auparavant nous désirons manifester notre accord avec l'une des premières remarques formulées par monsieur Dion: la réalité syndicale dépasse largement les effectifs syndicaux. On insistera jamais assez sur cette idée tant il est vrai qu'on ne peut attribuer de zone d'influence précise au mouvement syndical en le réduisant à des pourcentages, si raffinés puissent être leurs bases de compilation. Nous sommes tellement conscients de cette limite inhérente à ce type d'exercice que l'un des auteurs, dans un premier article publié sur cette question, en a fait explicitement état: «Les taux de syndicalisme nous renseignent sur l'évolution et l'importance numérique d'un phénomène institutionnel dont la dynamique dépasse les cadres du dénombrement pur et simple». ${ }^{1}$

\section{PREMIER ÉLÉMENT: LES CAUSES DE LA STAGNATION DU SYNDICALISME}

Pour expliquer la stagnation, voire la régression des taux du syndicalisme au Québec, monsieur Dion suggère de considérer, à titre d'hypothèse, les orientations et les attitudes de certaines centrales syn-

* MM. DELORME et LASSONDE sont tous deux agents de recherche au ministère du Travail et de la Main-d'oeuvre et au ministère des Affaires Sociales respectivement. Le point de vue qu'ils exposent ici ne reflète pas nécessairement celui des ministères auxquels ils sont rattachés.

1 LASSONDE, G., "Les taux du syndicalisme au Québec», Travail Québec, Vol. 13, no 2 , mars 1977 , pp. $25-28$, à la page 25 . 
dicales. On pourrait certes avoir recours à une hypothèse exploratoire de cette nature pour tenter d'expliquer les mouvements d'allégeance observés au niveau des effectifs syndicaux en général et plus particulièrement, pour essayer de rendre compte de la croissance des effectifs représentés par des syndicats indépendants. Cependant, il nous apparaît très difficile d'utiliser une telle hypothèse pour éclairer le phénomène du recul des effectifs syndicaux alors même qu'au Québec, la diversité des centrales et des affiliations possibles donne une dimension réelle au concept du pluralisme syndical. L'explication des orientations et des attitudes syndicales en regard de la diminution des effectifs syndicaux vaudrait peut-être pour le cas des États-Unis où le choix des affiliations est beaucoup plus restreint: ou bien l'on fait partie d'une union affiliée à la grande centrale FAT-COI, ou bien l'on est en présence d'un syndicat indépendant, même si ce dernier peut être numériquement important.

En contrepartie, d'autres hypothèses peuvent être avancées en guise de facteurs explicatifs de la stagnation numérique du syndicalisme québécois. Outre le cadre juridique de l'accréditation, nous en proposons deux anciennes: la résistance de certains employeurs à l'avènement du syndicalisme ainsi que certaines conséquences engendrées par le pluralisme syndical, particulièr ement lors de l'organisation de nouvelles unités. La résistance de certains employeurs à l'avènement du syndicalisme ne tient pas du mythe, bien au contraire; sans disposer de chiffres valables ni d'indices de mesure «scientifiques», nous savons que plusieurs employeurs s'opposent catégoriquement à l'implantation du syndicalisme au sein de leur entreprise, ce qui freine considérablement ses possibilités de croissance. Dans un mémoire conjoint présenté en 1977 au gouvernement québécois, les centrales C.E.Q. et C.S.N., confrontées quotidiennement avec la réalité des relations de travail, rapportent ce qui suit: «De nombreux employeurs, particulièrement dans les moyennes et petites entreprises, opposent une résistance active à l'exercice par leurs employés, du droit d'association. Un employé qui compte bien souvent des années de loyaux services est congédié sous des prétextes fallacieux, mais en fait c'est qu'il participe à la mise sur pied d'un syndicat». ${ }^{2}$

Le pluralisme syndical s'accompagne évidemment de la concurrence intersyndicale, laquelle prend beaucoup de relief à l'occasion de l'organisation de nouvelle unités que deux ou plusieurs syndicats se disputent. Cet aspect du pluralisme syndical, outre qu'il entraîne des délais dans l'émission des certificats d'accréditation, absorbe beaucoup d'énergie de la part des organisations syndicales dont l'employeur peut parfois tirer parti en appuyant sur la division des travailleurs relativement à leur allégeance. Dans une affaire récente ${ }^{3}$ par exemple, trois syndicats cherchent à obtenir le

2 Centrale de l'Enseignement du Québec et Confédération des Syndicats Nationaux, Mémoire commun de revendications présenté par la C.S.N. et la C.E.Q. au gouvernement du Québec, 28 février 1977, $60 \mathrm{pp}$., à la page 10.

3 Syndicat des employés de Coq d'Or Marieville (C.S.N.) et Construction and Supply Drivers and Allied Workers Teamsters, local 903 et local 1999, Routiers, Brasseries, Liqueurs douces et ouvriers de diverses industries et les Produits Coq d'Or Ltée, Bureau du Commissaire général du Travail, dossiers M-16374-1 à M-16374-5, René Gosselin, Commissaire du Travail, Montréal, le 5 juillet 1978, décision non rapportée. 
monopole de représentation des travailleurs; les requêtes en accréditation ont été successivement déposées en janvier, mars et mai 1978; constatant que les trois requérants possèdent la majorité requise pour être accrédités (sic), le Commissaire du travail n'a pas d'autre alternative que d'ordonner un vote d'allégeance, conformément à l'article 25 du Code du travail.

Qui soutiendra qu'une telle situation, fruit de la concurrence intersyndicale, favorise la syndicalisation, en songeant aux délais requis pour déterminer la représentativité des requérants ainsi qu'aux conséquences, pour les organisations syndicales, d'un vote à peu près égal en faveur des trois syndicats concernés? Nous émettons l'hypothèse que de telles situations, si elles ne posent pas d'obstacle formel à la croissance du syndicalisme, risquent d'en freiner l'élan.

SECOND ÉLÉMENT: LA PLACE DE LA F.T.Q. SUR L'ÉCHIQUIER SYNDICAL QUÉBÉCOIS

Monsieur Dion affirme, à propos des membres québécois du C.T.C., que seulement 46,7\% d'entre eux sont membres de la F.T.Q. Il cite, pour appuyer son assertion, notre propre texte, à la page 14 précisément. Nous soulignons d'abord que le texte mentionne $49,7 \%$ au lieu de $46,7 \%$, ce qui nous ramène à $50 \%$ des effectifs environ. De plus, cette proportion est nettement sous-estimée et il est faux de parler de 49 ou de $50 \%$. Pourquoi? La note infrapaginale 16 de la page 14 indique explicitement qu'une décision administrative de la F.T.Q. a amputé - au moment de référence de la cueillette des données - ses effectifs globaux de 51800 cotisants. Il aurait peut-être fallu ajouter que cette décision revêtait un caractère temporaire et que, sur une base annuelle, on comptabilisait en 1976, quelque 258956 membres de la F.T.Q., ce qui représentait une proportion de $62 \%$ des effectifs québécois du C.T.C., et non 46,7\%.

Quant aux tableaux de notre publication qui présentent la répartition des effectifs syndicaux selon l'affiliation par secteur d'activité économique et par région administrative, monsieur Dion nous fait grief d'avoir accolé la mention F.T.Q. aux colonnes C.T.C. et F.A.T.-C.O.I.-C.T.C. Même si nous disposions de statistiques établies sur une base annuelle des effectifs de la F.T.Q., le problème resterait entier quant à nous puisque questionner la pertinence de la mention F.T.Q. dans les tableaux VII et VIII de notre publication pose tout le problème de la place occupée par la F.T.Q. sur l'échiquier syndical québécois. S'il est vrai qu'au sens strict du terme, la F.T.Q. ne peut être considérée comme centrale syndicale au même titre que le C.T.C., il serait encore plus inexact, dans le contexte sociologique du Québec, de la reléguer au rang de fédération provinciale, organe du C.T.C. sur le plan horizontal. En plus de l'histoire même de la F.T.Q. qui traduit, pour partie, un désir d'autonomie toujours plus grand ${ }^{4}$, nous ne signalerons

4 Sur l'évolution historique de l'idéologie de la F.T.Q., voir: L. ROBACK, "L'idéologie de la Fédération des Travailleurs du Québec, évolution historique», texte non publié et présenté dans le cadre d'un séminaire sur le Canada français, 27 décembre 1967, 23 pp. 
ici que deux faits qui témoignent de la place qu'occupe la F.T.Q. sur la "carte» syndicale québécoise:

a) on se rappellera d'abord que la F.T.Q. était désignée comme telle, au titre d'association représentative, dans la Loi sur les relations de travail dans l'industrie de la construction, S.Q., 1968, c.45, article 5;

b) on notera aussi qu'à partir de 1974, le C.T.C. a délégué à la F.T.Q. sa juridiction sur les conseils de travail et sur l'éducation syndicale 5 , et ce à l'égard de tous les membres québécois du C.T.C.

Ces deux éléments tendent à démontrer que l'action de la F.T.Q. ne se limite pas à la représentation des intérêts des travailleurs auprès des pouvoirs publics, ce qui mérite bien, à notre avis, de faire mention de la F.T.Q. quand on parle du C.T.C. dans le cas du Québec.

\section{TROISIÈME ÉLÉMENT: LA MAGIE DES CHIFFRES OU LA CRAINTE SALUTAIRE QU'ILS PEUVENT INSPIRER}

Nous sommes les premiers à admettre le caractère relatif des chiffres portant sur l'état de la syndicalisation; s'il faut reconnaître qu'ils expriment des ordres de grandeur, nous trouvons exagérée l'hypothèse de monsieur Dion selon laquelle les chiffres publiés comportent des biais ou des marges d'erreur de l'ordre de 20 à $30 \%$. Lorsque tel écart, en provenance de la même source, se présente, le M.T.M.O. procède habituellement à des vérifications systématiques. Le problème est beaucoup plus difficile à trancher lorsqu'on compare diverses sources de renseignements. L'exemple de la Fédération des Affaires Sociales (C.S.N.), rapportée par monsieur Dion, s'avère ici très révélateur. Qu'en est-il au juste? Les données obtenues auprès de la fédération en vertu de la Loi sur les déclarations des corporations et des syndicats ouvriers, S.R.C., 1970, c. C-31, lui confèrent en 1976 des effectifs globaux de 49193 (voir notre texte en page 34); comparant cette donnée avec le nombre de 71990 publié par la C.S.N. dans un numéro de Travail Québec ${ }^{6}$, monsieur Dion s'étonne à bon droit de l'écart substantiel entre ces deux données. Pour tenter de trouver la meilleure approximation possible de la réalité, nous avons, de notre côté, utilisé deux sources complémentaires. Le fichier C.S.N. des cotisants par syndicat attribue à la F.A.S., sur la base d'une moyenne arithmétique annuelle, un nombre de 55330 adhérents en 1976. Une source patronale ${ }^{7}$ indique, pour la même

5 Fédération des Travailleurs du Québec, Rapport du secrétaire général, 15e congrès, 28 novembre au 2 décembre 1977, 63 pp., à la page 13.

6 RIOUX, M., "Confédération des Syndicats Nationaux: faire avancer les choses dans le sens d'une dignité humaine toujours à reconquérir», Travail Québec, Vol. 14, no 2, septembre 1978, pp. 9-12, à la page 11 .

7 Ministère des Affaires Sociales, Direction générale des relations de travail, Statistiques concernant les salariés de niveau syndicable du secteur des Affaires Sociales, période du ler janvier 1976 au 31 décembre 1976, novembre 1978, 36 pp., à la page 8. 
période de référence, un nombre de 54150 syndiqués affiliés à la C.S.N.; ce dernier chiffre est exprimé en termes d'employés «équivalent temps plein», pour utiliser une expression du jargon technique en usage dans le secteur des Affaires sociales. Notons toutefois que les effectifs rapportés par le M.A.S. incluent les syndiqués C.S.N. affiliés à la Fédération des Affaires Sociales autant que ceux regroupés dans la Fédération des professionnels salariés et cadres du Québec. Quoiqu'il en soit, le chiffre rapporté dans notre publication, soit 49 193, constitue une approximation sous-estimant probablement les effectifs réels de la F.A.S. mais qui demeure fort convenable lorsqu'on le compare avec ceux utilisés dans deux autres sources. Dans cette valse des chiffres, il y aurait fort à parier qu'une source additionnelle de renseignements apporterait un éclairage différent, c'est-à-dire un cinquième chiffre... Le moins qu'on puisse dire, c'est que l'absence d'uniformité et de cohérence entre les sources de renseignements interdit au lecteur de manifester trop de sûreté dans l'interprétation de ce type de données.

\section{QUATRIÈME ÉLÉMENT: QUELQUES AMÉLIORATIONS À APPORTER}

Dans la conclusion de sa recension, monsieur Dion suggère d'apporter aux publications éventuelles deux améliorations précises: fournir des taux sectoriels de syndicalisation suivant le sexe de la main-d'oeuvre salariée ainsi que des données numériques sur la population couverte par les conventions collectives signées en vertu du Code québécois du travail. Si intéressante qu'elle puisse être, la première suggestion n'est pas réalisable à court terme pour la simple et bonne raison que Statistique Canada ne fournit pas, dans la publication mensuelle La population active ${ }^{8}$, de répartition de la main-d'oeuvre féminine par secteur d'activité économique qui soit suffisamment décomposée. C'est ainsi, à titre d'exemple, que les industries manufacturières sont toutes regroupées ensemble, ne permettant pas ainsi de procéder à des ventilations suffisamment précises. Les données du recensement pourraient peut-être être utilisées dans la mesure où les divisions, pour les activités économiques, seraient compatibles avec celles en usage pour la compilation des autres tableaux. Resterait évidemment à résoudre le problème de la continuité des données d'une année à llautre.

La seconde suggestion formulée par monsieur Dion est plus facile à réaliser mais elle recèle, en revanche, des limites sérieuses dans le contexte d'une étude quantitative sur la syndicalisation. Il s'agit d'abord d'une donnée statique, à tout le moins sur une période de 3 ans, quand ce n'est pas pour une longue période; il arrive dans certains cas, - et nous avons pu le constater à quelques reprises -, que le nombre d'employés couverts par la convention collective soit le même depuis le moment où l'on a effectué la première observation, c'est-à-dire depuis l'accréditation! C'est dire à quel point le problème du vieillissement de l'information trouve ici sa pleine mesure. En second lieu, les chiffres qu'on pourrait éventuellement fournir

8 Voir par exemple, Statistique Canada, La population active, cat. no 70-001, décembre 1976, tableau 48, p. 61: «Population active selon la province, la branche d'activité et le sexe». 
ne tiennent pas compte des syndicats nouvellement accrédités et qui négocient une première convention collective, non plus que des conventions collectives non déposées au ministère. Nous ignorons l'ampleur de ce dernier phénomène mais quelques indices, notamment des sentences arbitrales de griefs pour lesquels nous ne pouvons retracer de convention collective au ministère, permettent de conclure à son existence. Ne serait-ce pas un moyen ingénieux d'éviter de donner prise, en temps opportun, à tout maraudage jugé indésirable?

\section{ECOLE DE RELATIONS INDUSTRIELLES DE L'UNIVERSITÉ DE MONTRÉAL}

\section{Colloques}

- Formes nouvelles de syndicalisme, (5e colloque), 1974, 66 pages.

- L'État et la transformation des relations industrielles au Québec, (6e colloque), 1976, 64 pages.

- Les conflits en milieux de travail, (7e colloque), 1977, 100 pages.

- Pour une meilleure qualité de vie ... les avantages sociaux, (8e colloque), 1978, 96 pages.

- La réforme des lois du travail, (9e colloque), 1979, 124 pages.

\section{Monographies}

- C. D'AOUST et L. LECLERC, La jurisprudence arbitrale québécoise en matière de congédiement, 1978, 181 pages.

- L.P. DAUBIGNEY, Salaire et marché du travail interne à l'entreprise, 1978, 51 pages.

- G. GUERIN, Le système de planification des ressources humaines dans l'entreprise, 1978,95 pages. 